\title{
Antioxidant Capacity in Vanilla Extracts Obtained by Applying Focused Microwaves
}

\author{
Adalith Rojas-López, María P. Cañizares-Macías* \\ Departamento de Química Analítica, Facultad de Química, Universidad Nacional Autónoma de México, México D.F., México. \\ Email: *pilarm@unam.mx
}

Received April $6^{\text {th }}, 2013$; revised May $6^{\text {th }}, 2013$; accepted May $13^{\text {th }}, 2013$

Copyright (C) 2013 Adalith Rojas-López, María P. Cañizares-Macías. This is an open access article distributed under the Creative Commons Attribution License, which permits unrestricted use, distribution, and reproduction in any medium, provided the original work is properly cited.

\begin{abstract}
ORAC method and a continuous flow injection method based on Folin-Ciocalteau reaction (FI-FC) were used for determining the antioxidant activity in extracts obtained by using focused microwaves. Analysis of the antioxidant capacity (AC) of the main compounds of vanilla (vanillin, p-hydroxybenzaldehyde, p-hydroxybenzoic acid and vanillic acid) was also carried out. Vanilla extracts obtained by using focused microwaves had a higher AC (between $72 \%$ and $117 \%$ ) than the obtained by conventional methods. Vanillin had a linear correlation with the antioxidant capacity of the extracts and it is the most influential compound in the antioxidant power. The AC calculated by the ORAC method and the FI-FC method had a ratio 2:1 because of different kinetics and reaction mechanisms of the antioxidants with the reagents, so it is necessary more than one method to establish the antioxidant power in food. On base on the results of the present study microwaves energy can be used to obtain vanilla extracts to improve the $\mathrm{AC}$ of them.
\end{abstract}

Keywords: Focused Microwaves; Vanilla; Antioxidant Capacity; ORAC Method; Flow Injection; Folin-Ciocalteau

\section{Introduction}

The early news from vanilla are in the middle 15 th century, when Aztecs conquered the Totonaca Empire who used vanilla to make a drink named "xocolatl" (chocolate). Libellus de Medicinalibus Indorum Herbis, Cruz Balbiano codex, were the first document where vanilla is named with its Nahuatl name: "tlilxochitl" (black flower) [1]. For three centuries Mexico was the only producer of vanilla in the world. In 1841, Edmond Albins, a farmer from Reunion Island had the idea of pollinating the flower with a bamboo stick. From then vanilla began to spread through Africa and Asia [2].

Vanilla is the only orchid that produces an important commercial fruit for its flavour and smell. There are more than 110 species of vanilla, but the Vanilla planifolia, also known as Vanilla fragans, is the most important source of the natural commercial vanilla $[1,3,4]$.

Vanilla has more than 250 compounds but only 26 have concentrations higher than $1 \mathrm{mg} \cdot \mathrm{kg}^{-1}$. Among non-volatiles compounds of vanilla are: tannins, polyphenols, free amino acids and resins and among volatiles compounds are: carbonyl aromatic and aliphatic alcohols, aromatic

\footnotetext{
"Corresponding author.
}

acids, aromatic esters, phenols, lactones, aliphatic and aromatic hydrocarbons, terpenoids, etc. Vanillin (1\% - 3\%), vanillic acid $(0.1 \%-0.2 \%)$, p-hydroxy-benzaldehyde $(0.1 \%-0.2 \%)$ and p-hydroxybenzoic acid $(0.01 \%$ $0.02 \%$ ) are the most important phenolics compounds produced during cured process [5-7].

Antioxidants have beneficial effects on the conservation of the food, avoiding their oxidation, besides having healthy effects; so industries of food, pharmaceutical and cosmetic have been used antioxidants to give better advantage over yours products. The main synthetic antioxidants used by the industry are: propyl gallate, butylated hydroxy anisole, hydroxyl toluenehydroxyanisole, tertiary butylhydroquinone, most of them are not very healthy, therefore natural antioxidants instead of the synthetic compounds have been more used [8-10]. Plants and spices are the main target to look for natural antioxidants. There are some studies to identify the compounds that should act as antioxidants [11]. Among phytochemicals best characterized in vegetable food polyphenols have been the most studied due to their high dairy ingest [12]. Moreover, polyphenols are able to fix other reactive compounds as $\mathrm{HO} \cdot \mathrm{NO}_{2}^{\bullet}, \mathrm{N}_{2} \mathrm{O}_{3}, \mathrm{ONOOH}$ and $\mathrm{HOCl}$ and also are able to fix metallic ions (specially iron and cop- 
per) to obtain poorly active compounds in the formation of reactive species [13].

Inhibition of the chain reaction, oxygen captation, inhibition of singlet oxygen, metals quelation and inhibition of oxidative enzymes are mechanism that makes very complex the antioxidant action in the food. Therefore, several analytical methods are necessary to get a precise evaluation of the antioxidant capacity (AC) [14]. Based on the chemical reaction involved, two categories of the main antioxidant capacity tests can be named:

a) Tests based on the hydrogen atom transfer reaction: these methods measure the competitive kinetic reaction and the quantification comes from the kinetic curves. Usually these are made from a free radicals synthetic generator, an oxidative molecular probe and an antioxidant. The Oxygen Radical Absorbance Capacity (ORAC) test is one of the more used [15-17].

b) Tests based on the electron transfer reaction: in these methods a redox reaction is carried out and the reduction of the oxidant marks the finish of the reaction. The reaction with Folin-Ciocalteau reagent is one of these. This method is principally used to determine total polyphenols $[18,19]$.

In the last decade, there has been an increasing demand for new extraction techniques enabling automation, shortening extraction times and reduction of organic solvents consumption [20,21]. Advances in preparation of solid samples have brought a great number of new techniques such as focused microwave-assisted energy [2224]. Microwaves have been used for different chemical process accelerating digestion [25] and organic and inorganic synthesis [26-28]. Focused microwaves have been also used successfully to extract vanillin from vanilla beans increasing the efficiency at $100 \%$, so it has been possible to accelerate extraction procedures of different compounds without oxidation of them when the conditions of extraction are controlled [29].

Vanilla is one of the species more consumed, mainly in extracts, with a high antioxidant capacity (AC). Vanillin is its main compound and the majority phenol. In this paper the antioxidant capacity in ethanolic vanilla extracts obtained by applying of focused microwaves was measured. A maceration procedure [30] was also carried out and the results were compared. Vanillin concentration was determined and a study about the relation between vanillin and $\mathrm{AC}$ was carried out. $\mathrm{AC}$ was determined by two tests way: a) ORAC method, using fluorescein as probeand 2,2'-azobis(2-methylpropionamidine) dihydrochlorideas radicals' generator and b) a flow injection method using Folin-Ciocalteau reagent (FI-FC).

\section{Material and Methods}

\subsection{Reagents and Solutions}

All reagents were analytical grade and used water was distilled.

Fluorescein (Sigma-Aldrich) and 2,2'-azobis(2-methylpropionamidine) dihydrochloride, 97\% (AAPH) from Sigma-Aldrich were used by the ORAC method. A fluorescein stock solution $\left(10 \mu \mathrm{g} \cdot \mathrm{mL}^{-1}\right)$ was prepared in a $0.05 \mathrm{M} \mathrm{pH} 7$ phosphates buffer. $0.03 \mu \mathrm{g} \cdot \mathrm{mL}^{-1}$ fluorescein solutions to obtain the kinetics curves were prepared from the stock solution using the same buffer. A $0.22 \mathrm{M}$ AAPH solution was also used. A 1:10 dilution from $\mathrm{Fo}_{-}$ lin-Ciocalteau reagent (Merck) and a $0.5 \mathrm{M} \mathrm{NaOH}$ (Baker) solution were used by the FI-FC method. Caffeic acid (Sigma-Aldrich) was used as reference to determine the AC by both methods.

Standard solutions obtained from a $1000 \mu \mathrm{g} \cdot \mathrm{mL}^{-1}$ vanillin (Sigma-Aldrich) stock solution were used to build the vanillin calibration curve. Vanillic acid, p-hydroxybenzaldehyde and p-hydroxybenzoic acid were also purchased by Sigma-Aldrich.

A 70:30 ethanol (Baker): water mixture was also used to obtain the vanilla extracts.

\subsection{Instruments}

A fluorimeter PMT-FL Fialab and a thermostated bath Lab-line 1800 were used to determine the $\mathrm{AC}$ by the ORAC method. A peristaltic pump (Gilson minipuls), an injection valve (Rheodyne), Teflon and Tygon tubing, a Hellma $18 \mu \mathrm{L}$ internal volume flow cell and a Cary UVVis spectrophotometer (Varian) as detector were used to build the FI manifold. A FI manifold reported by Valdéz and Cañizares [7] was used to determine vanillin.

A $300 \mathrm{~W}$ maximum power focused microwaves oven (Prolabo) was used to obtain the vanilla extracts. An ultrasonic bath (Branson Model 2510R), a centrifuge (HETTICH EBA 20) and a pHmeter (Oakton) were also used. A controlled temperature oven (Rios Rocha) was used for the moisture assay.

\subsection{Samples}

All vanilla samples were Mexican. Two different cured vanilla beans quality were analyzed: a) gourmet quality, from Papantla, Veracruz: $15 \mathrm{~cm}$ length, dark brown colour and glossy beans; b) second quality (named zacatillo) from Ayotoxco, Puebla, used to make handicrafts: from $11 \mathrm{~cm}$ to $17 \mathrm{~cm}$ length, light brown colour with clear stripes beans. Green beans from Papantla, Veracruz were also used.

Gourmet vanilla was purchased in a local market from Papantla. A Cooperative of Women from Ayotoxco, Puebla, who cultivate, harvest and cure vanilla, gave the $\mathrm{Za}$ catillo vanilla beans.

For the analyses a stock of $500 \mathrm{~g}$ of each kind of vanilla beans was used. Beans were cut to a size of $2 \mathrm{~mm} \times$ $2 \mathrm{~mm}$ and homogenized. They were stored in refrigera- 
tion until were used.

Vanilla green beans from Ayotoxco were also analyzed. The beans were collected and stored at $4^{\circ} \mathrm{C}$ until analysis.

\subsection{Getting of the Extracts}

Focused microwaves-assisted extraction (FMAE) [29]: 1 $\mathrm{g}$ of cut vanilla beans $(2 \times 2 \mathrm{~mm})$ was poured into a 10 $\mathrm{cm}$ test tube of $3 \mathrm{~cm}$ of internal diameter, which was placed into a water bath (test tube of $4.5 \mathrm{~cm}$ of internal diameter). $25 \mathrm{ml}$ of a $70 \%(\mathrm{v} / \mathrm{v})$ ethanol-water solution were added to the sample. A refrigerant was adapted to the test tube to avoid loss of the extractant and a $150 \mathrm{~W}$ microwaves irradiation power was applied to the sample. Twenty cycles of $1 \mathrm{~min}$ irradiation each one with a delay time between them of 3 min were carried out. The total extraction time was $80 \mathrm{~min}$. Once the extraction was over, solution was filtered and water was added until reaching a final volume of $100 \mathrm{~mL}$.

Maceration extraction (ME) [29,30]: $1 \mathrm{~g}$ of cut vanilla beans $(2 \times 2 \mathrm{~mm})$ was poured into a $10 \mathrm{ml}$ volumetric flask covered with $2 \mathrm{ml}$ of ethanol and $1 \mathrm{ml}$ of water. The solution was macerated for 12 hours. $2 \mathrm{ml}$ of ethanol were added mixing well all the content. Maceration continued for 3 days. The solution was drained funnel dry, packing solids firmly and percolating slowly with a 50\% ethanol solution until reaching a final volume of $10 \mathrm{ml}$.

\subsection{Determination of the Antioxidant Capacity (AC) in Extracts of Vanilla Beans}

\subsubsection{ORAC Method}

It was used, with some modifications, for the determination the $\mathrm{AC}$ in the vanilla extracts obtained from the vanilla beans. $2 \mathrm{~mL}$ of the $0.03 \mu \mathrm{g} \cdot \mathrm{mL}^{-1}$ fluorescein (FL) solution, $2 \mathrm{~mL}$ of blank (phosphates buffer $(0.5 \mathrm{M}, \mathrm{pH} 7)$ and $1 \mathrm{~mL}$ of standard (caffeic acid) or sample (diluted 1:1000) were set into a $13 \times 100 \mathrm{~cm}$ testing tube. During $15 \mathrm{~min}$ were incubated at $37^{\circ} \mathrm{C}$. Later $1 \mathrm{~mL}$ of AAPH was added and then the fluorescence intensity was measured setting this moment as cero time $\left(t_{0}\right)$. After $5 \mathrm{~min}$ of a new incubation step the signal was measured again. This procedure was repeated until fluorescence intensity decayed at $20 \%$ from initial value. The fluorescence intensity was measured $\lambda \mathrm{ex}=486 \mathrm{~nm}$ and $\lambda \mathrm{em}=500 \mathrm{~nm}$. The measurements were carried out in triplicate for each sample and standard.

A calibration curve was obtained by plotting the total area under the curve (AUC) against caffeic acid concentrations in the $1 \mu \mathrm{g} \cdot \mathrm{mL}^{-1}-6 \mu \mathrm{g} \cdot \mathrm{mL}^{-1}$ range. The area under the kinetic curve (AUC) was calculated for each standard and from these values the total area under the kinetic curve $\left(\mathrm{AUC}_{\text {Total }}\right)$. In Figure 1 the integration ranges and the fluorescence intensity decreasing curve are shown. The trapezoid method according to Equation (1) was used to calculate the AUC;

$$
\begin{aligned}
\mathrm{AUC}= & \left(\frac{\left(y_{0}+y_{5}\right)}{2} \times 5\right) \\
& +\left(\frac{\left(y_{5}+y_{10}\right)}{2} \times 5\right)+\ldots+\left(\frac{y_{n-5}+y_{n}}{2} \times 5\right)
\end{aligned}
$$

where $y_{0}$ is the initial fluorescence signal and $y_{5}, y_{10}$, $y_{n-5}, \cdot, y_{n}$ are the fluorescence intensity values at different incubation time: $5,10, n-5, \cdots, n$ minutes.

$\mathrm{AUC}_{\text {Total }}$ was solved from Equation (2):

$$
\mathrm{AUC}_{\text {Total }}=\mathrm{AUC}_{\mathrm{ANTIOXIDANT}}-\mathrm{AUC}_{\mathrm{BLANK}}
$$

\subsubsection{FI-FC Method}

This method has been also used to determine AC en different kind of food. FI methods are faster than batch method and it has been proved that they are very precise. Folin-Ciocalteau reagent has been used for phenolic compounds analysis both by FI [31] and by batch methods obtained a very good precision [19,32]. In Figure 2 the used FI manifold is shown. $100 \mu \mathrm{L}$ of sample or caf-

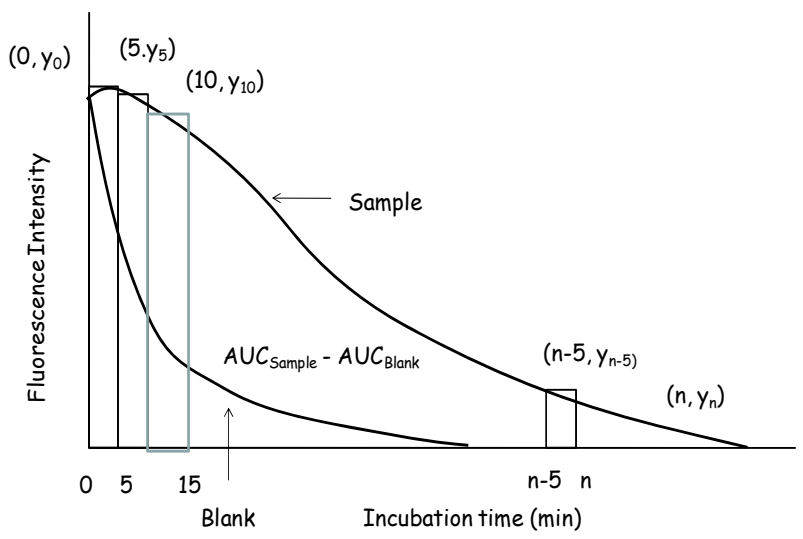

Figure 1. Trapezoid method (area under the curve (AUC)) to determine the antioxidant capacity (AC) using the ORAC method.

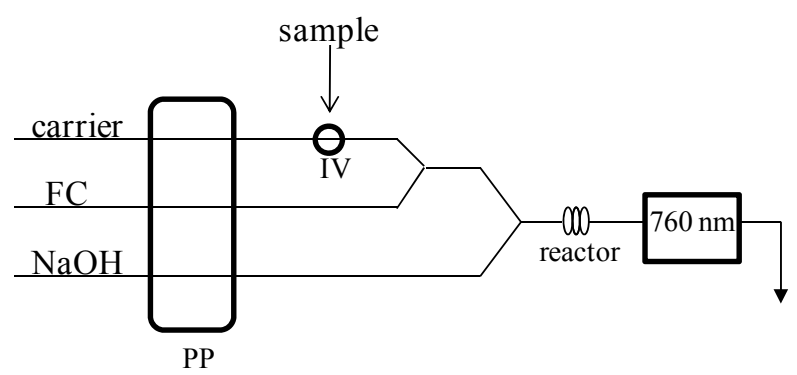

Figure 2. Flow injection manifold to determine the antioxidant capacity (AC) using the Folin-Ciocalteau reagent (FIFC). PP: peristaltic pump; IV: injection valve; FC: FolinCiocalteau reagent $(\mathbf{1 0 \%})$; carrier: distilled water; $\mathrm{NaOH}$ : $0.5 \mathrm{M}$; reactor: $100 \mathrm{~cm}$. 
feic acid standard were injected into the carrier (distilled water) to merge with Folin-Ciocalteau reagent and later with a $0.5 \mathrm{M} \mathrm{NaOH}$ stream. The formed complex was measured at $760 \mathrm{~nm}$. Extracts were diluted 1:500 to measure the AC in the vanilla.

The precisions of the methods were calculated by determination of the antioxidant capacity of six independent aliquots of the sample for each evaluated method.

\subsubsection{Determination of Vanillin}

A flow injection manifold was used to quantify vanillin in accordance with Valdez and Cañizares [7]. $100 \mu \mathrm{l}$ of sample were injected into a carrier of $0.01 \mathrm{~N} \mathrm{NaOH}$, which carries the sample to the reactor where the vanillin was hydrolyzed. Finally, the hydrolyzed plug passed through a flow cell located in the UV-VIS spectrophotometer, where the product was measured at $347 \mathrm{~nm}$.

\subsection{Determination of Moisture in Cured Vanilla Beans}

The procedure was carried out in accordance with the established in the American Society for Testing and Material [33]. From one and five grams of the sample were weighed and placed into a previously dried container, which was covered and then weighed again. Later, the stopper was removed from the container and both were placed in the oven. The sample was dried for $4 \mathrm{~h}$ at $105^{\circ} \mathrm{C} \pm 3^{\circ} \mathrm{C}$. At the end of the specified period, the container was quickly covered and placed in a desiccator during $1 \mathrm{~h}$. After this time the sample was weighed. The drying and weighing procedures were repeated until the mass loss between two successive weightings was not more than $0.005 \mathrm{~g}$ (or until the specimen showed a gain in mass). The moisture was calculated by using the following equation:

$$
\text { Moisture content }=\left[\frac{(M-D)}{(M-T)}\right] \times 100
$$

where:

$M=$ original mass of the specimen and weighing bottle

$D=$ oven-dry mass of the specimen

$T=$ mass of the empty weighing bottle

\section{Results and Discussion}

\subsection{Characteristics of the Quantification Methods}

It has been widely accepted that the radical system used for the antioxidant evaluation may influence the experimental results [34]. There are many methods of analysis to determine the antioxidant capacity and radical scavenging activity in food (iron(II) chelating activity, total antioxidant capacity, DPPH assays and lipid peroxidation) and a number of different reagents have been used. Each one of these methods has different values of AC depending on the kind of reaction and the analytical measurement (fluorescence, UV-Vis, electrochemical, etc.) [35]. Although Trolox is the most used reagent by ORAC method due to its high sensibility, caffeic acid has been also used because it has a high antioxidant power [36]. So, in this paper all results have been expressed as $\mathrm{mg}$ caffeic $\mathrm{acid} / \mathrm{g}$ of vanilla bean.

\subsubsection{ORAC Method}

The calibration curve was built from the caffeic acid kinetics graphics. In Figure $\mathbf{3}$ is showed some signals of three different concentrations of caffeic acid; some signals of vanilla extracts obtained by both extraction methods are also showed. For blank, initial fluorescence intensity falls down quickly because there is not an antioxidant and the peroxiles radicals generated by thermic decomposition of AAPH reacts with fluorescein obtaining a non-fluorescent product. If the reaction is carried out with an antioxidant the fall is not so quick therefore when the caffeic acid concentration increases the lag phase is larger, because caffeic acid fixes the peroxil radicals.

The linear range was from $1 \mu \mathrm{g} \cdot \mathrm{mL}^{-1}$ to $6 \mu \mathrm{g} \cdot \mathrm{mL}^{-1}$ with a lineal equation $\mathrm{AUC}_{\text {Total }}=3589.7( \pm 179.70)$ [caffeic acid] $+1809.8( \pm 727.74)$ and a regression coefficient of 0.9962 . At concentration higher than $6 \mu \mathrm{g} \cdot \mathrm{mL}^{-1}$ the decay of the fluorescence intensity in $20 \%$ was too long.

The detection limit (DL) was evaluated on the standard deviation of the response of the blank and the slope using the ratio $3.3 \mathrm{~s} / \mathrm{m}$, where $s$ is the standard deviation of the response of the blank and $m$ is the slope of the calibration curve of the analyte. The detection limit was $0.81 \mu \mathrm{g} \cdot \mathrm{mL}^{-1}$ and the quantification limit using the ratio $10 \mathrm{~s} / \mathrm{m}$ was $1.65 \mu \mathrm{g} \cdot \mathrm{mL}^{-1}$.

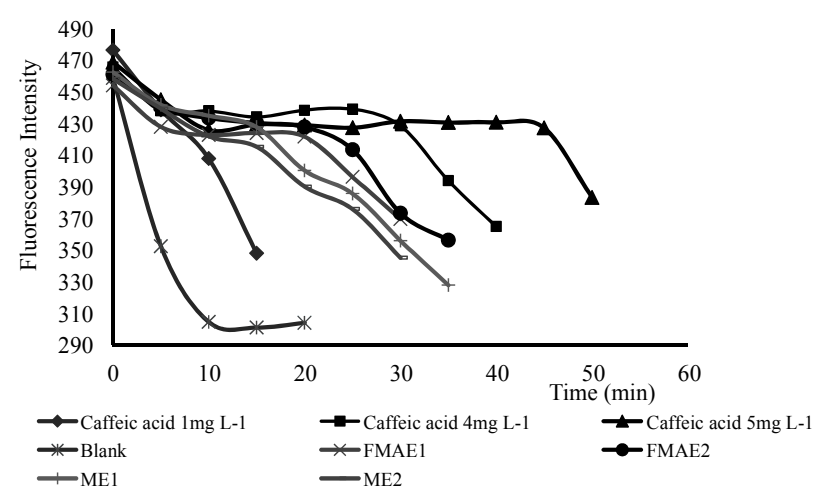

Figure 3. Plot of fluorescence intensity signal vs time for three caffeic acid standard solutions and for two vanilla extracts obtained by focused microwaves assisted extraction $\left(\mathrm{FMAE}_{1}\right.$ and $\left.\mathrm{FMAE}_{2}\right)$ and by maceration extraction $\left(\mathrm{ME}_{1}\right.$ and $\mathrm{ME}_{2}$ ). 


\subsubsection{FI-FC Method}

The linear equation $\mathrm{Abs}=0.011( \pm 0.0001)$ [caffeic acid] $+0.0085( \pm 0.0058)$ was obtained for a caffeic acid concentration range between $5 \mu \mathrm{g} \cdot \mathrm{mL}^{-1}$ and $100 \mu \mathrm{g} \cdot \mathrm{mL}^{-1}$. The regression coefficient was 0.9998 , the detection limit of $3.55 \mu \mathrm{g} \cdot \mathrm{mL}^{-1}$ and the quantification limit of 6.35 $\mu \mathrm{g} \cdot \mathrm{mL}^{-1}$.

\subsection{Determination of Vanillin in Extracts}

Extracts obtained by both methods were between light and medium brown color (depending of the kind of bean), with a vanilla characteristic odor. When focused microwaves were used the vanillin amount in the extracts obtained from both kinds of beans increased. Once again it was demonstrated that when focused microwaves are applied the vanillin extraction from vanilla beans increases: for zacatillo beans between $75 \%$ and $87 \%$ and for gourmet beans between $81 \%$ and $93 \%$, comparing with the obtained results by the official method (ME). For green beans the vanillin concentration was too low therefore this was only determined when microwaves were used. The results are showed in Table 1. In this same table moisture of the beans is also showed. Moisture is a quality control parameter for vanilla beans and it has to be between $25 \%$ and $30 \%$ for gourmet vanilla and between 20 and $24 \%$ for vanilla of second quality [30]. The evaluated beans were in these classifications. Although the vanilla concentration was higher when moisture in the vanilla beans was higher, it was not possible to conclude that moisture higher increases the vanillin concentration in the vanilla extracts. On the other hand, in a previous paper was demonstrated that when cured vanilla beans were dried at $135^{\circ} \mathrm{C}$ for $4 \mathrm{~h}$, the vanilla concentration decreased between $30 \%$ and $40 \%$ [29]. The waves of microwave are non-ionizant radiations that cause the mobility of the molecules due to the migration of ions and the bipolar rotation. When the electric camp decreases the thermic disorder that produce energy as heat is restored. This law makes that the interaction between the analytes in a matrix and the extractant is better and the extraction is more efficient [22]. There are some examples of focused microwave extraction that improve the extraction of polar and non-polar compounds in different matrixes as the extraction of ketoprofen in pharmaceutical cream formulation [37], the extraction of heterocyclic amines in meat [38] or in order to clarify the possible hazard of acidic pharmaceuticals (ibuprofen, naproxen, ketoprofen, and diclofenac) in river water and sediments [39]. Using microwave in solid samples allows more efficient and faster sample treatment, so it is being more used in analysis laboratories. Microwaves are non-destructive energy, so can be applied in food under controlled radiation which has allowed to be used, for example to accelerate the oxidation process in olive oil [40].
On the other hand, several studies have demonstrated that vanillin helps to the stability in the beans avoiding oxidation, but it is necessary good conservation conditions.

\subsection{Antioxidant Capacity (AC) in Vanilla Beans}

ORAC method is the most used method to measure the $\mathrm{AC}$ in food because of its high sensitivity [15,41]. Nevertheless there are a number of methods to determine the $\mathrm{AC}$ where the found values are very different between methods. For this reason, usually more than one method is used to evaluate the $\mathrm{AC}$ in food $[42,43]$.

Vanilla, specie, is mainly used as flavoring and it is used in a very low amount in our daily diet but it could help to get the daily recommended antioxidants. So, it is important to develop extraction methods of improving of the antioxidant power in the extracts, such as the focused microwaves assisted extraction method evaluated in this research. In Figure 3 is showed the signals obtained by the ORAC method of different vanilla extracts obtained by the maceration method (ME) and by the FMAE method. The signals of vanilla extracts show a large lag phase which is indicative of a high AC but they are lower when the extracts are obtained by the ME. The extracts analyzed by the FI-FC method had values of AC lower than by the ORAC method although by both methods the $\mathrm{AC}$ increased when microwaves were applied. The results of the AC obtained by both methods are showed in Table 2. The AC for gourmet beans was higher than for zacatillo beans between a $55 \%$ and a $57 \%$, using both

Table 1. Concentration of vanillin in extracts obtained by focused microwaves (FMAE) and by maceration (ME). It is also shown the moisture for each kind of bean.

\begin{tabular}{|c|c|c|c|}
\hline \multirow{2}{*}{ Sample } & \multicolumn{2}{|c|}{ Vanillin concentration $(\%, w / w)$} & \multirow[t]{2}{*}{ Moisture (\%) } \\
\hline & $\mathrm{ME}$ & FMAE & \\
\hline Zacatillo & & & $24.70 \pm 0.20$ \\
\hline 1 & $0.62 \pm 0.012$ & $1.16 \pm 0.03$ & \\
\hline 2 & $0.57 \pm 0.025$ & $1.08 \pm 0.02$ & \\
\hline 3 & $0.65 \pm 0.015$ & $1.14 \pm 0.03$ & \\
\hline Gourmet & & & $27.80 \pm 0.24$ \\
\hline 1 & $1.07 \pm 0.020$ & $2.02 \pm 0.02$ & \\
\hline 2 & $0.95 \pm 0.015$ & $1.81 \pm 0.02$ & \\
\hline 3 & $1.05 \pm 0.031$ & $1.96 \pm 0.03$ & \\
\hline Green beans & nd & $0.27 \pm 0.01$ & - \\
\hline
\end{tabular}

nd: no detected. 
Table 2. Values of the antioxidant capacity (AC) by the ORAC method and by the Flow Injection-Folin Ciocalteau (FI-FC) method for vanilla extracts obtained by focused microwave (FMAE) and by maceration (ME).

\begin{tabular}{ccccc}
\hline \multirow{2}{*}{ Sample } & \multicolumn{4}{c}{ AC (expressed as mg caffeic acid g ${ }^{-1}$ bean) } \\
\cline { 2 - 5 } & \multicolumn{2}{c}{ ORAC } & \multicolumn{2}{c}{ FI-FC } \\
\cline { 2 - 5 } FMAE & ME & FMAE & ME \\
\hline Gourmet & & & & \\
1 & $77.22 \pm 1.03$ & $37.86 \pm 1.65$ & $35.74 \pm 0.30$ & $20.32 \pm 0.13$ \\
2 & $69.93 \pm 1.10$ & $33.69 \pm 1.42$ & $32.33 \pm 0.95$ & $18.37 \pm 0.36$ \\
3 & $75.13 \pm 0.85$ & $37.17 \pm 2.05$ & $34.77 \pm 0.65$ & $19.99 \pm 0.44$ \\
Zacatillo & & & & \\
1 & $45.09 \pm 0.87$ & $24.74 \pm 0.74$ & $24.03 \pm 0.16$ & $12.18 \pm 0.22$ \\
2 & $44.58 \pm 0.78$ & $20.52 \pm 0.84$ & $20.48 \pm 0.56$ & $12.20 \pm 0.42$ \\
3 & $46.66 \pm 1.00$ & $23.27 \pm 1.25$ & $21.61 \pm 0.34$ & $12.52 \pm 0.16$ \\
Green & $6.70 \pm 0.32$ & nd & nd & nd \\
\hline
\end{tabular}

nd. no detected.

analysis methods. Also, when the vanillin concentration increased the $\mathrm{AC}$ does, so the highest $\mathrm{AC}$ was for extracts obtained using focused microwaves because they had an amount of vanillin higher than when the maceration method was used. The AC:vanillin ratio by the ORAC method was between $35: 1$ and $41: 1$ and by the FI-FC method was between $17: 1$ and $21: 1$, with an excellent correlation coefficient between vanillin concentration and AC: of 0.994 for the ORAC method and of 0.996 for the FI-FC method (Figure 4). The AC by the ORAC method increased between $82 \%$ and $117 \%$ in the extracts obtained by focused microwaves in comparison with the obtained by the maceration method; by the FIFC method the increased was between $72 \%$ and $97 \%$. The results show that the $\mathrm{AC}$ value is higher when microwaves are applied independently of the analysis method used.

F test was calculated to compare the standard deviation from the two methods of extraction using the two analysis methods, ORAC and FI-FC. The F test showed that there was no difference in the variances of the methods because experimental value (1.56) was lower than critical value (4.026). So the precision was similar by both methods. So $t$ test was calculated based on equal variance. The null hypothesis was: the $\mathrm{AC}$ is the same by both methods, FMAE and ME. The results showed that the values of the media were statistically different, so the $\mathrm{AC}$ is higher when FMAE was used to obtain vanilla extracts, $t$ value was higher than the critical value $\left(t_{9}=\right.$ 2.26).

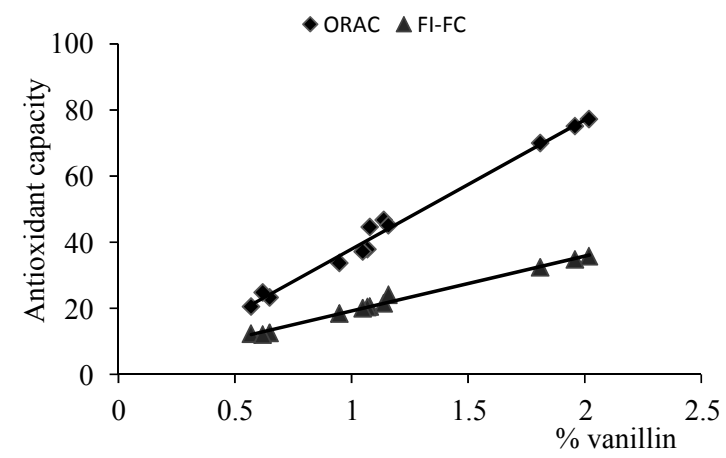

Figure 4. Correlation between the vanilla concentration and the ORAC method ( $\bullet$ ) and the FI-FC method (A). Antioxidant capacity expressed as mg caffeic acid $\mathrm{g}$ bean $^{-1}$.

The vanillin concentration in green beans was very low and therefore the $\mathrm{AC}$, so it is shown the importance of the vanillin in the in antioxidant capacity in cured vanilla beans. In green beans, vanillin is fixed to glucose as glucovanillin and its antioxidant power is not strong enough so green vanilla beans have a very poor AC.

On the other hand, the $\mathrm{AC}$ value is different between an analysis method and another one for a same compound, so when caffeic acid was used as standard, which is a strong antioxidant, the vanilla $\mathrm{AC}$ values by the ORAC method were approximately two times higher than by the FI-FC method (Table 2) but by both methods of analysis the AC increased or decreased in the same proportion for the same extracts. This difference is due to that vanillin is majority compound of vanilla and the analytical response is not the same for the two methods. The linear correlation between the ORAC and FI-FC methods was 0.99. Different electrochemical methods for the determination of antioxidant power in food have been compared with DPPH and ABTS radical cation assays by Buratti et al. $[42,44]$. The antioxidant power values are different but the linear correlation among them have been higher than 0.92 .

So, a calibration curve using vanillin as standard to react with the Folin-Ciocalteau was evaluated. The calibration curve had a slope of $0.0081 \mathrm{mg} \cdot \mathrm{mL}^{-1}$ (1.36 times lower than the slope for caffeic acid). For at same concentration (expressed as $\mathrm{mmol} \cdot \mathrm{L}^{-1}$ ) the analytical signal of vanillin was lower than for caffeic acid.

The AC of vanillin was also measure using the ORAC method and was compared with others compounds of the vanilla beans.

\subsection{Antioxidant Capacity of Vanillin, p-Hydroxybenzaldehyde (PHB), Vanillic Acid and p-Hydroxibenzoic Acid (HBA) by ORAC Method}

Besides vanillin the vanilla beans have others compounds which are extracted at the same time of vanillin. The 
most important are p-HBA, vanillic acid and HBA. Vanillin is between 10 and 30 times more concentrated than PHB and vanillic acid and the ratio increases for HBA between 100 and 140 times.

Standards of PHB, HBA and vanillic acid to concentrations closed at the vanillin concentration in the diluted extracts were tested. The vanillin concentration in the extracts obtained by focused microwaves from zacatillo beans was of $467 \mathrm{mg} \cdot \mathrm{L}^{-1}$ so it was diluted 1000 times and $\mathrm{AC}$ was determined, the vanillin concentration was $0.50 \mathrm{mg} \cdot \mathrm{L}^{-1}$ approximately. The others compounds were analyzed at this same concentration. At $0.5 \mathrm{mg} \cdot \mathrm{L}^{-1}$ of each compound the AC could be only measured for vanillin and for vanillic acid, obtaining $2.3 \mathrm{mg}$ caffeic acid $\mathrm{L}^{-1}$ and $0.53 \mathrm{mg}$ caffeic acid $\mathrm{L}^{-1}$, respectability. The $\mathrm{AC}$ of vanillin is 4.3 times higher than vanillic acid. PHB and HBA did not show antioxidant capacity. This behavior is because the antioxidant activity of phenolics acids and their esters depends on the number of hydroxyl groups in the molecule that would be strengthened by steric hindrance [45]. The electron withdrawing properties of the carboxylate group in benzoic acids has a negative influence on the H-donating abilities of the hydroxyl benzoates. The monohydroxy benzoic acids show no antioxidant activity in the ortho and para positions in terms of hydrogen-donating capacity against radical generated in the aqueous phase but the $m$-hydroxy acid has antioxidant activity. This is consistent with the electron withdrawing potential of the single carboxyl functional group on the phenol ring affecting the $o$ - and $p$-positions. The monohydroxy benzoates are, however, effective hydroxyl radical scavengers, due to their propensity to hydroxylation and the high reactivity of the hydroxyl radical. With a methylene group between the phenolic ring and the carboxylate group, as in the vanillin, the $o$ - and $m$-hydroxy derivatives have high antioxidant activities. Carried out a study of AC of vanillin using different assays were carried out by Akiro Tai et al. [46]. They showed that vanillin had a stronger antioxidant capacity than ascorbic acid and Trolox by the ORAC method but it showed no activity in the DPPH radical and galvinoxyl radical scavenging assays. By the FI-FC method a $5 \mathrm{mg} \cdot \mathrm{L}^{-1}$ concentration of each analyte was used. At this concentration only vanillin and vanillic acid showed AC where the vanillin concentration was 2.43 times higher than the vanillic acid concentration $\left(4.94 \mathrm{mg}\right.$ caffeic acid $\mathrm{L}^{-1}$ vs $2.03 \mathrm{mg}$ caffeic acid $\mathrm{L}^{-1}$ ). The $\mathrm{AC}$ for $500 \mathrm{mg} \cdot \mathrm{L}^{-1} \mathrm{PHB}$ and HBA solutions was $4.53 \mathrm{mg}$ caffeic acid $\mathrm{L}^{-1}$ and $35.7 \mathrm{mg}$ caffeic acid $L^{-1}$, respectively. So, the analysis of $\mathrm{AC}$ using different method is suggested to assure the antioxidant properties from a compound.

\section{Conclusions}

The AC value was different among analysis methods so it is convenient to use more than one method to establish the antioxidant power of the food. In this paper is shown that the vanilla has a high AC independently of used method, but the obtained numeric values were different. So, when caffeic acid is used as standard, which is a strong antioxidant, the vanilla $\mathrm{AC}$ values by the ORAC method were approximately two times higher than by the FI-FC method but by both methods of analysis the AC increased or decreased in the same proportion for the same extracts. The linear correlation between the ORAC and FIFC methods was 0.99 . Also the antioxidant power values reported by Buratti et al. $[42,44]$ were different but the linear correlation among them was higher than 0.92 .

When microwaves were applied the AC in the extracts increased between $72 \%$ and $117 \%$ with a strong relationship with the vanillin concentration. The vanillin concentration in the obtained extracts by using microwaves increased between $75 \%$ and $93 \%$. So, it has been showed that when microwaves are applied to obtain natural vanilla extracts the AC increased because the vanillin concentration is higher and it is the most influential compound in the antioxidant power.

Vanilla, specie, is mainly used as flavoring and it is used in a very low amount in our daily diet but it could help to get the daily recommended antioxidants. So, it is important to develop extraction methods of improving of the antioxidant power in the extracts, such as the focused microwaves assisted extraction method used. On the other hand, several studies have demonstrated that vanillin helps to the stability in the beans avoiding oxidation, but it is necessary good conservation conditions.

The results showed that the vanilla $\mathrm{AC}$ is given, principally, for the vanillin amount in the beans and in the extracts. They also showed that green vanilla beans have a very poor AC.

\section{Acknowledgements}

The Faculty of Chemistry and the Programa de Apoyo a Proyectos de Investigación e Innovación Tecnológica, PAPIIT (grant No. IT202812-3) of the Universidad NacionalAutónoma de México are gratefully acknowledged for financial support. The Ayotoxco vanilla beans were supplied by the Ayotoxco farmers with the support of the InstitutoPoblano de Desarrollo Rural A.C.

\section{REFERENCES}

[1] E. Hágsater, M. A. Soto-Arenas, G. A. Salazar-Chávez, R. Jiménez Machorro, M. A. López-Rosas and R. L. Dressler, "Las Orquídeas de México," Instituto Chinoín A.C., Redacta S.A. de C.V., Mexico City, 2005.

[2] S. R. Rao and G. A. Ravishankar, "Vanilla Flavour: Production by Conventional and Biotechnological Routes," Journal of the Science of Food and Agriculture, Vol. 80, No. 3, 
2000, pp. 289-304

doi:10.1002/1097-0010(200002)80:3<289::AID-JSFA543 $>3.0 . \mathrm{CO} ; 2-2$

[3] A. K. Sinha, U. K. Sharma and N. Sharma, "A Comprehensive Review on Vanilla Flavor: Extraction, Isolation and Quantification of Vanillin and Others Constituents," International Journal Food Science and Nutrition, Vol. 59, No. 4, 2008, pp. 299-326. doi:10.1080/09687630701539350

[4] M. J. Dignum, J. Kerler and R. Verpoorte, "Vanilla Production: Technological, Chemical and Biosynthetic Aspects," Food Reviews International, Vol. 17, No. 2, 2001, pp. 199-219. doi:10.1081/FRI-100000269

[5] A. K. Sinha, S. C. Verma and N. Sharma, "Development and Validation of an RP-HPLC Method for Quantitative Determination of Vanillin and Related Phenolic Compounds in Vanilla Planifolia," Journal of Separation Science, Vol. 30, No. 1, 2007, pp. 15-20. doi:10.1002/jssc. 200600193

[6] E. Odoux, J. Escoute and J. Verdell, "The Relation between Glucovanillin, $\beta$-D-Lucosidase Activity and Cellular Compartmentation during the Senescence, Freezing and Traditional Curing of Vanilla Beans," Annals Applied Biology, Vol. 149, No. 1, 2006, pp. 43-52. doi:10.1111/j.1744-7348.2006.00071.x

[7] C. Valdez-Flores and M. P. Cañizares-Macías, "On-Line Dilution and Detection of Vanillin in Vanilla Extracts Obtained by Ultrasound," Food Chemistry, Vol. 105, No. 3, 2007, pp. 1201-1208.

doi:10.1016/j.foodchem.2007.02.028

[8] S. P. Wong, L. P. Leong and J. Koh, "Antioxidant Activities of Aqueous Extracts of Selected Plants," Food Chemistry, Vol. 99, No. 4, 2006, pp. 775-783. doi:10.1016/j.foodchem.2005.07.058

[9] N. V. Yanishlieva, E. Marinova and J. Pokorny, "Natural Antioxidant from Herbs and Spices," European Journal of Lipid Science and Technology, Vol. 108, No. 9, 2006, pp. 776-793. doi:10.1002/ejlt.200600127

[10] Pastene, M. Gómez, H. Speisky and L. Nuñez-Vergara, "Un Sistema Para la Detección de Antioxidantes Volátiles Comúnmente Emitidos Desde Especias y Hierbas Medicinales," Quimi Nova, Vol. 32, No. 2, 2009, pp. 482-487. doi:10.1590/S0100-40422009000200035

[11] J. C. Espín, M. T. García-Conesa and F. A. Tomás-Barberán, "Nutraceuticals: Facts and Fiction," Phytochemical, Vol. 68, No. 22, 2007, pp. 2986-3008. doi:10.1016/j.phytochem.2007.09.014

[12] M. E. Drago-Serrano, M. López-López and T. R. SainzEspuñes, "Componentes Bioactivos de Alimentos Funcionales de Origen Vegetal," Revista Mexicana de Ciencias Farmacéuticas, Vol. 37, No. 1, 2006, pp. 58-68.

[13] B. Halliwell and J. M. Gutteridge, "Free Radicals in Biology and Medicine," 3rd Edition, Oxford University Press, Oxford, 1999.

[14] E. N. Frankel and A. S. Meyer, "The Problems of Using One-Dimensional Methods to Evaluate Multifunctional Food and Biological Antioxidants," Journal of Science Food Agriculture, Vol. 80, No. 13, 2000, pp. 1925-1941. doi:10.1002/1097-0010(200010)80:13<1925::AID-JSFA7

\section{$14>3.0 . \mathrm{CO} ; 2-4$}

[15] R. H. Bisby, R. Brooke and S. Navaratnam, "Effect of Antioxidant Oxidation Potential in the Oxygen Radical Absorption Capacity (ORAC) Assay," Food Chemistry, Vol. 108, No. 3, 2008, pp. 1002-1007. doi:10.1016/j.foodchem.2007.12.012

[16] B. Ou, M. Hampsch-Woodill and R. L. Prior, "Development and Validation of an Improved Oxygen Radical Absorbance Capacity Using Fluorescein as the Fluorescent Probe," Journal of Agriculture and Food Chemistry, Vol. 49, No. 10, 2001, pp. 4619-4626. doi:10.1021/jf010586o

[17] A. Zulueta, M. J. Esteve and A. Frígola, "ORAC and TEAC Assays Comparison to Measure the Antioxidant Capacity of Food Products," Food Chemistry, Vol. 114, No. 1, 2009, pp. 310-316. doi:10.1016/j.foodchem.2008.09.033

[18] M. Celeste, C. Tomás, A. Cladera, J. M. Estela and V. Cerdà, "Enhanced Automatic Flow Injection Determination of the Total Polyphenol Number of Wines Using the Folin-Ciocalteau Reagent," Analytica Chimica Acta, Vol. 269, No. 1, 1992, pp. 21-28. doi:10.1016/0003-2670(92)85128-S

[19] V. L. Singleton, R. Orthofer and R. M. Lamuela-Raventós, "Analysis of Total Phenols and Other Oxidation Substrates and Antioxidants by Means of Folin-Ciocalteu Reagent," Methods in Enzymology, Vol. 299, No. 14, 1999, pp. 152-178. doi:10.1016/S0076-6879(99)99017-1

[20] D. Sterbova, D. Matejícek, J. Vlcek and V. Kubán, “Combined Microwave-Assisted Isolation and Solid-Phase Purification Procedures Prior to Chromatographic Determination of Phenolic Compounds in Plant Materials," Analytica Chimica Acta, Vol. 513, No. 2, 2004, pp. 435-444. doi:10.1016/j.aca.2004.03.031

[21] Y. Zuo, L. Zhang, J. Wu, J. W. Fritz, S. Medeiros and C. Rego, "Ultrasonic Extraction and Capillary Gas Chromatography Determination of Nicotine in Pharmaceutical Formulations," Analytica Chimica Acta, Vol. 526, No. 1, 2004, pp. 35-39. doi:10.1016/j.aca.2004.09.035

[22] J. L. Luque-García and M. D. Luque de Castro, "Where Is Microwave-Based Analytical Equipment for Solid Simple Pre-Treatment Going?" Trends Analytical Chemistry, Vol. 22, No. 2, 2003, pp. 90-98. doi:10.1016/S0165-9936(03)00202-4

[23] J. L. Luque-García, J. Velasco, M. C. Dobarganes and M. D. Luque de Castro, "Fast Quality Monitoring of Oil from Prefried and Fried Foods by Focused Microwave-Assisted Soxhlet Extraction," Food Chemistry, Vol. 76, No. 2, 2002, pp. 241-248. doi:10.1016/S0308-8146(01)00293-X

[24] E. E. Stashenko, B. E. Jaramillo and J. R. Martínez, "Comparison of Different Extraction Methods for the Analysis of Volatile Secondary Metabolites of Lippia Alba (Mill.) N. E. Brown, Grown in Colombia and Evaluation of Its in Vitro Antioxidant Activity," Journal of Chromatography $A$, Vol. 1025, No. 1, 2004, pp. 93-103. doi:10.1016/i.chroma.2003.10.058

[25] K. J. Lamble and S. J. Hill, "Microwave Digestion Procedures for Environmental Matrices," Analyst, Vol. 123, No. 7, 1998, pp. 103R-133R. doi:10.1039/a800776d

[26] S. Vadahanambi, S. J. H. Jung and I. K. Oh, "Microwave Syntheses of Graphene and Graphene Decorated with Me- 
tal Nanoparticles," Carbon, Vol. 49, No. 13, 2011, pp. 44494457. doi:10.1016/j.carbon.2011.06.038

[27] S. Mishra, G. Sen, G. U. Rani and S. Sinha, "Microwave Assisted Synthesis of Polyacrylamide Grafted Agar (Agg-PAM) and Its Application as Flocculant for Wastewater Treatment," International Journal of Biological Macromolecules, Vol. 49, No. 4, 2011, pp. 591-598. doi:10.1016/j.ijbiomac.2011.06.015

[28] S. V. Jadhav, E. Suresh and H. C. Bajaj, "Microwave-Assisted Solvent-Free Synthesis of $\alpha, \alpha$ '-Bis(substituted benzylidine)cycloalkanones Catalyzed by $\mathrm{SO}_{4} 2-/ \mathrm{ZrO}_{2}$ and $\mathrm{B}_{2} \mathrm{O}_{3} / \mathrm{ZrO}_{2}$," Green Chemistry Letters Review, Vol. 4, No. 3, 2011, pp. 249-256. doi:10.1080/17518253.2010.546371

[29] A. Longares-Patrón and M. P. Cañizares-Macías, "Focused Microwaves-Assisted Extraction and Simultaneous Spectrophotometric Determination of Vanillin and $p$-Hydroxybenzaldehyde from Vanilla Fragans," Talanta, Vol. 69, No. 4, 2006, pp. 882-887. doi:10.1016/j.talanta.2005.11.030

[30] Mexican Norm: NMX-FF-074-SCFI-2009, "Productos No Industrializados Para Uso Humano-Vainilla (Vanilla fragrans (Salisbury) Ames Especificaciones y Métodos de Prueba. Non Industrialized Food Products for Human Consumption-Vanilla (Vanilla fragrans (Salisbury) Ames). Specifications and Test Methods," Secretaría de Comercio y Finanzas, Mexico City, 2009.

[31] M. P. Cañizares, M. T. Tena and M. D. Luque de Castro, "On Line Coupling of a Liquid-Liquid Extraction FlowReversal System to a Spectrophotometric Flow-Through Sensor for the Determination of Polyphenols in Olive Oil," Analytica Chimica Acta, Vol. 323, No. 1-3, 1996, pp. 55-62.

[32] T. Ercetin, F. S. Senol, I. Erdogan, I. Orhan and G. Toker, "Comparative Assessment of Antioxidant and Cholinesterase Inhibitory Properties of the Marigold Extracts from Calendula arvensis L. and Calendula officinalis L.," Industrial Crops Products, Vol. 36, No. 1, 2012, pp. 203208.

[33] American Society for Testing and Material, "Annual Book of ASTM,” ASTM D 1348-94, Vol. 6.03, 2003, pp. 287292.

[34] L. Yu, J. Perret, B. Davy, J. Wilson and C. L. Melby, "Free Radical Scavenging Properties of Wheat Extracts," Journal of Food Science, Vol. 67, No. 22, 2002, pp. 26002603. doi:10.1111/j.1365-2621.2002.tb08784.x

[35] E. A. Abdelilah, K. Hajar and H. Abdellatif, "Phenolic Profile and Antioxidant Activities of Olive Mill Wastewater," Food Chemistry, Vol. 132, No. 1, 2012, pp. 406412. doi:10.1016/j.foodchem.2011.11.013

[36] M. F. Barroso, C. Delerue-Matos and M. B. P. P. Oliveira, "Electrochemical Evaluation of Total Antioxidant Capacity of Beverages Using a Purine-Biosensor," Food Chemistry, Vol. 132, No. 2, 2012, pp. 1055-1062. doi:10.1016/j.foodchem.2011.10.072

[37] S. Labbozzetta, L. Valvo, P. Bertochi, S. Alimunti, M. C.
Gaudiano and L. Manna, "Focused Microwave-Assisted Extraction and LC Determination of Ketoprofen in the Presence of Preservatives in a Pharmaceutical Cream Formulation," Chromatographia, Vol. 69, No. 3, 2009, pp. 365-368. doi:10.1365/s10337-008-0892-Z

[38] A. Martín-Calero, V. Pino, J. H. Ayala, V. González and A. M. Alfonso, "Ionic Liquids as Mobile Phase Additives in High-Performance Liquid Chromatography with Electrochemical Detection: Application to the Determination of Heterocyclic Aromatic Amines in Meat-Based Infant Foods," Talanta, Vol. 79, No. 3, 2009, pp. 590-597. doi:10.1016/j.talanta.2009.04.032

[39] M. Varga, J. Dobor, A. Helenkár, L. Jurecska, J. Yao and G. Záray, "Investigation of Acidic Pharmaceuticals in River Water and Sediment by Microwave-Assisted Extraction and Gas Chromatography-Mass Spectrometry," Microchemica Journal, Vol. 95, No. 2, 2010, pp. 353-358. doi:10.1016/j.microc.2010.02.010

[40] M. P. Cañizares-Macías, J. A. García-Mesa and M. D. Luque de Castro, "Determination of Oxidative Stability on Olive Oil Using Focused-Microwave Energy to Accelerate the Oxidation Process," Analytical and Bioanalytical Chemistry, Vol. 378, No. 2, 2004, pp. 479-483. doi:10.1007/s00216-003-2280-8

[41] D. P. Singh, J. Beloy, J. K. McInerney and L. Day, "Impact of Boron, Calcium and Genetic Factors on Vitamin C, Carotenoids, Phenolic Acids, Anthocyanins and Antioxidant Capacity of Carrots (Daucus carota)," Food Chemistry, Vol. 132, No. 3, 2012, pp. 1161-1170. doi:10.1016/j.foodchem.2011.11.045

[42] S. Buratii, N. Pelligrini, O. Brenna and S. Mannino, "Rapid Electrochemical Method for the Evaluation of the Antioxidant Power of Some Lipophilic Food Extracts," Journal of Agriculture and Food Chemistry, Vol. 49, No. 11, 2001, pp. 5136-5141. doi:10.1021/jf010731y

[43] D. Lettieri-Barbato, D. Villaño, B. Beheydt, F. Guadagni, I. Trogh and M. Serafini, "Effect of Ingestion of Dark Chocolates with Similar Lipid Composition and Different Cocoa Content on Antioxidant and Lipid Status in Healthy Humans," Food Chemistry, Vol. 132, No. 3, 2012, pp. 1305-1310. doi:10.1016/j.foodchem.2011.11.109

[44] S. Buratti, S. Benedetti and M. S. Cosio, "Evaluation of the Antioxidant Power of Honey, Propolis and Royal Jelly by Amperometric Flow Injection Analysis," Talanta, Vol. 71, No. 3, 2007, pp. 1387-1392. doi:10.1016/j.talanta.2006.07.006

[45] C. A. Rice-Evans, N. J. Miller and G. Paganga, "Structure Antioxidant Activity Relationships of Flavonoids and Phenolic Acids," Free Radical Biology and Medicine, Vol. 20, No. 7, 1996, pp. 933-956. doi:10.1016/0891-5849(95)02227-9

[46] A. Tai, T. Sawano, S. F. Yasama and H. Ito, "Evaluation of Antioxidant Activity of Vanillin by Using Multiple Antioxidant Assays," Biochemica and Biophysica Acta, Vol. 1810, No. 2, 2011, pp. 170-177. doi:10.1016/j.bbagen.2010.11.004 


\section{Abreviations}

AC: Antioxidant Capacity

AAPH: 2,2'-azobis(2-methylpropionamidine) dihydrochloride

AUC: Area under Curve

FI-FC: Flow Injection-Folin Ciocalteau method
FMAE: Focused Microwaves-Assisted Extraction HBA: p-hydroxybenzoic acid

ME: Maceration Extraction

ORAC: Oxygen Radical Absorbance Capacity

PHB: p-hydroxybenzaldehyde 